\author{
Irena SOBOL, Dariusz KACPRZAK, Witold KOSIŃSKI ${ }^{1}$
}

\title{
OPTIMIZING OF A COMPANY'S COST UNDER FUZZY DATA AND OPTIMAL ORDERS UNDER DYNAMIC CONDITIONS ${ }^{2}$
}

\begin{abstract}
Summary
The purpose of this article is to suggest tools of inventory management which would determine economically optimal order quantities. One of them is based on the so-called fixed order quantity model which takes into account several elements of inventory cost, such as ordering cost, transportation and storing cost, frozen capital cost, as well as extra discounts. The tool is based on fuzzy concepts represented by Ordered Fuzzy Numbers. The second tool takes into account the dynamics and works on the basis of replenishment system. This tool can be treated as a kind of controller. Examples of using this tools are presented.
\end{abstract}

Key words: Ordered Fuzzy Numbers, defuzzification, Economic Order Quantity Model, optimal order quantity

\section{Introduction}

The main objective of a good inventory management system is to keep the inventory cost at the minimum level. There are several elements of inventory cost, such as ordering cost, transportation cost, frozen capital cost, cost of loss (i.e. aging), cost of lost sales due to inventory shortages, and others. Several inventory models have been built based on the above. There are two most commonly used inventory models: fixed order quantity system and replenishment system.

In the first system, the quantity to be ordered is fixed and re-orders are made once the stock reaches a certain pre-determined level called safety stock. It means that the next order is typically fixed and based on the average consumption during the lead time plus some safety stock. Often in calculation the buffer stock is the one-day inventory consumption.

Under the second system, the quantity to be ordered is not fixed, the next order is decided based on the lead time of the material, maximum stock level, i.e. the ordered level changes with time.

\footnotetext{
${ }^{1}$ Irena Sobol, Department of Computer Science, Polish-Japanese Institute of Information Technology, e-mail: s8531@pjwstk.edu.pl; Dariusz Kacprzak, PhD, Faculty of Computer Science, Bialystok University of Technology, e-mail: d.kacprzak@pb.edu.pl.

2 This work was supported by Bialystok University of Technology grant S/WI/2/2011.
} 
In this paper we propose two tools of inventory management. The first one is based on the fixed order quantity model which takes into account several elements of inventory cost. The tool based on fuzzy concepts represented by Ordered Fuzzy Numbers. The second tool takes into account the dynamics, and works on the basis of replenishment system. This tool can be treated as a kind of controller.

When dealing with the first tool the fuzzy optimization problem for the total cost function is formulated within a space, where all variables of the model are fuzzy. After choosing of a particular defuzzification functional, an appropriate theorem is formulated and gives a solution to the fuzzy optimization problem.

When developing the second tool, the authors faced a situation where material demand is irregular during the production process. This results in no equal ordered levels as well as in different elapse times between orders.

The organization of the paper is as follows. In Section 2, within the economic order quantity model the problem of supply management is considered, in which the optimal size of a delivery from outside is determined; this minimizes total costs, when crisp unit costs of purchase, transportation and storage are given. Then in Section 3, a fuzzy optimization problem is formulated together with its solution and a numerical example. In Section 4, a problem of management of supply and determining an optimal size of a delivery from outside is considered while the material demand depends on time. Then a solution algorithm is described. The final results of this section is a kind of a controller together with a numerical example. In Section 5, conclusions are formulated. Appendix refers to the model of Ordered Fuzzy Numbers.

\section{Economic order quantity model}

Inventory management within an enterprise is an integral part of its operating activities, as it influences its competitive advantage and its financial liquidity. The purpose of inventory management is to have the stock at a high enough level and operate smoothly, while incurring the lowest possible operating costs. The presented formulation is within the general framework of the model of economic order quantity (EOQ).

We consider an abstract inventory item. To estimate the cost of inventory management we formulate the main assumptions in the EOQ model:

1. the abstract inventory item is split into units;

2. we refer a defined time frame, say one year;

3. demand is constant in time;

4. sales is uniform in time and known;

5. the next delivery arrives when the stock is for one day only.

Let us start with deterministic formulation in which the following objects appears:

- $\quad D$ - annual inventory demand, measured in number of units;

- $\quad D / 360$ - daily demand for supply (assuming that a year has 360 days);

- $\quad Q$ - order quantity, measured in number of units;

- $\quad Q_{0}$ - daily consumption of inventory;

- $\quad D / Q-$ frequency of deliveries; 
- $\quad 360 /(D / Q)=t_{0}-$ time between successive deliveries;

- $\quad c_{p}$ - unit price of purchase;

- $\quad c_{t}-$ transportation cost of a single delivery;

- $\quad c_{S}$ - unit inventory cost per day;

- $\quad r(Q)$ - discount function on purchase;

- $\quad s(Q)-$ discount function on stored inventory;

- $\quad K(Q)$ - total cost;

- $\quad K_{p}$ - purchase cost;

- $\quad K_{f}-$ frozen capital cost;

- $\quad K_{t}$ - transportation (delivery) cost;

- $K_{S}$ - storage cost;

- $\quad R$ - banking interest rate, used to calculate the cost of frozen capital.

We can write the general expression of the total cost $K(Q)$, as the sum of the purchase $\operatorname{cost} K_{p}$, the frozen capital cost $K_{f}$, the transportation (delivery) cost $K_{t}$ and the storage cost $K_{s}$, i.e.

$$
K(Q)=K_{p}+K_{f}+K_{t}+K_{s} .
$$

Suppose that we get the discount $r(Q)$ on purchase and the discount $s(Q)$ on stored inventory depending on the amount of $Q$, both as step functions:

and

$$
r(Q)=\left\{\begin{array}{ccc}
r_{0}=0 & \text { if } & 0<Q<Q_{1}^{r} \\
r_{1} & \text { if } & Q_{1}^{r} \leq Q<Q_{2}^{r} \\
r_{2} & \text { if } & Q_{2}^{r} \leq Q \leq D
\end{array}\right.
$$

$$
s(Q)=\left\{\begin{array}{ccc}
s_{0}=0 & \text { if } & 0<Q<Q_{1}^{S} \\
s_{1} & \text { if } & Q_{1}^{S} \leq Q<Q_{2}^{S} \\
s_{2} & \text { if } & Q_{2}^{S} \leq Q \leq D
\end{array}\right.
$$

where $Q_{1}^{r}, Q_{2}^{r}, Q_{1}^{s}$ and $Q_{2}^{S}$ are fixed quantities (here 3 steps have been assumed, however, more steps can also be considered). The purchase cost $K_{p}$ depends on the quantity of the single delivery $Q$, the frequency of deliveries $D / Q$, the discount $r(Q)$ and the unit price $c_{p}$, and is given by

$$
K_{p}=c_{p} \cdot(1-r(Q)) \cdot Q \cdot \frac{D}{Q}=c_{p} \cdot(1-r(Q)) \cdot D .
$$

The cost of frozen capital depends on the number of deliveries $D / Q$, the money spent on a single delivery, the banking interest rate $R$, and on the single delivery $Q$. The form of the purchase cost $K_{p}$ gives the following cost $K_{f}$ of frozen capital:

$$
K_{f}=c_{p} \cdot(1-r(Q)) \cdot Q \cdot \frac{D}{Q} \cdot \frac{R}{\frac{D}{Q}}=c_{p} \cdot(1-r(Q)) \cdot Q \cdot R
$$

We can see that the expression $K_{f}$ represents a step function, which is piecewise linear. The cost of the transportation (delivery) $K_{t}$ depends on the annual frequency of deliveries $D / Q$ and the transportation cost of a single delivery $c_{t}$, i.e.

$$
K_{t}=c_{t} \cdot \frac{D}{Q}
$$


According to the assumptions 3 to 5 , the storage cost $K_{S}$ depends on the annual frequency of deliveries $D / Q$, the discount $s(Q)$, the unit inventory cost $c_{S}$ and the level of inventory between successive deliveries. The level of inventory is given by

$$
\int_{0}^{t_{0}}\left(-\frac{Q}{t_{0}} \cdot t+Q+Q_{0}\right) d t=\left(\frac{Q}{2}+Q_{0}\right) \cdot t_{0}
$$

and the storage cost by

$$
\begin{gathered}
K_{s}=\frac{D}{Q} \cdot c_{s} \cdot(1-s(Q)) \cdot\left(\frac{Q}{2}+Q_{0}\right) \cdot t_{0}=\frac{D}{Q} \cdot c_{s} \cdot(1-s(Q)) \cdot\left(\frac{Q}{2}+Q_{0}\right) \cdot \frac{360}{\frac{D}{Q}}= \\
=180 \cdot c_{s} \cdot(1-s(Q)) \cdot\left(Q+2 \cdot Q_{0}\right) .
\end{gathered}
$$

Hence the function describing the total cost $K(Q)$ in (1) has summed up to $K(Q)=c_{p} \cdot(1-r(Q)) \cdot(D+Q \cdot R)+c_{t} \cdot \frac{D}{Q}+180 \cdot c_{s} \cdot(1-s(Q)) \cdot\left(Q+2 \cdot Q_{0}\right) \cdot(9)$

FIGURE 1.

Graphical representation of discount functions and subintervals $L_{k}, k=$ $0,1,2,3,4$.

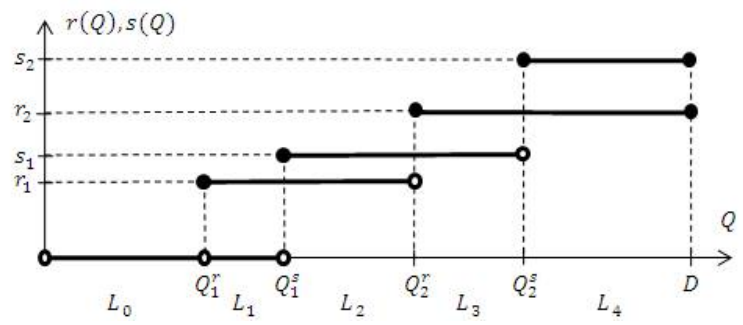

Source: own work.

The optimization problem of inventory management requires us to find the minimum of the cost function $K(Q)$. The argument which gives the minimum is the optimal value of the order quantity. Please note that in $K(Q)$ the first and the last component both depend on $Q$ in a piecewise way. Suppose that

$$
0<Q_{1}^{r}<Q_{1}^{S}<Q_{2}^{r}<Q_{2}^{S}<D
$$

The search for the optimal value should be performed in a piecewise way, i.e. considering each subinterval (Fig. 1.).

$$
L_{0}=\left(0, Q_{1}^{r}\right), L_{1}=\left[Q_{1}^{r}, Q_{1}^{s}\right), L_{2}=\left[Q_{1}^{s}, Q_{2}^{r}\right), L_{3}=\left[Q_{2}^{r}, Q_{2}^{s}\right), L_{4}=\left[Q_{2}^{s}, D\right] \text {. }
$$

Thus the global optimum is the quantity which gives the minimal cost over these five values calculated from each subinterval. Since

and

$$
\frac{\partial K(Q)}{\partial Q}=c_{p} \cdot(1-r(Q)) \cdot R-c_{t} \cdot \frac{D}{Q^{2}}+180 \cdot c_{s} \cdot(1-s(Q))
$$

$$
\frac{\partial K(Q)}{\partial Q}=0 \Leftrightarrow Q^{*}=\sqrt{\frac{c_{t} \cdot D}{c_{p} \cdot(1-r(Q)) \cdot R+180 \cdot c_{s} \cdot(1-s(Q))}}
$$

in each of these subintervals $L_{k}, k=0,1,2,3,4$ the local extreme is attained at 


$$
Q_{k}^{*}=\sqrt{\frac{c_{t} \cdot D}{c_{p} \cdot\left(1-r_{i}\right) \cdot R+180 \cdot c_{s} \cdot\left(1-s_{j}\right)}}
$$

where the following identification has been assumed between these sets of indexes:

$$
\begin{gathered}
0=k \leftrightarrow[i j]=[00], 1=k \leftrightarrow[i j]=[10], 2=k \leftrightarrow[i j]=[11], \\
3=k \leftrightarrow[i j]=[21], 4=k \leftrightarrow[i j]=[22] .
\end{gathered}
$$

If $Q_{k}^{*} \in L_{k}$, then the optimal value can appear in one of these subintervals or at their borders, i.e. it is attained at the argument given by

$$
Q_{\text {opt }}=\arg \min \left\{\left\{K\left(Q_{k}^{*}\right), k=0,1,2,3,4\right\}, K\left(Q_{1}^{r}\right), K\left(Q_{1}^{s}\right), K\left(Q_{2}^{r}\right), K\left(Q_{2}^{s}\right), K(D)\right\} .
$$

In the model (9) all components such as prices, discounts, total demand, have been assumed to be constant and known in advance. But in reality, many factors that influence economic decisions are not exactly known, and there is a margin of uncertainty. There are several options to handle this, like stochastic modelling, interval methods and, last but not least, the fuzzy approach sketched in this paper.

\section{Fuzzy optimization problem}

The present formulation is within the framework of the model of the Economic Order Quantity (EOQ) and similar to the one proposed in the set of Convex Fuzzy Numbers (CFN) by [Vuješević et al., 1996] and repeated by [Kuchta, 2001]. In the OFN's framework problems in economics and administrative accounting were formulated in [Chwastyk, Kosiński, 2013; Kosiński et al., 2013]. Our aim is to give a general solution to the optimization problem with the cost function given by (9) when $D, c_{p}, c_{t}$ and $c_{s}$ are fuzzy and represented by Ordered Fuzzy Numbers. It will be easy to see that the arithmetic of OFN manifests its superiority over the arithmetic of Convex Fuzzy Numbers, and the complex calculations performed by authors of [Kuchta, 2001; Vuješević et al., 1996] can be avoided. The only thing we need to do is choose the defuzzification functional which suits the decision maker the most. For more details on OFN we refer to in the Appendix.

Let $\Phi(\cdot)$ be the defuzzification functional chosen by the decision maker. Then the problem of minimizing the fuzzy cost $K(Q)$ gives us the economic order quantity. Writting it explicitly

$$
\text { find } \arg \{\min \Phi(K(Q)): Q \in \mathcal{R}\}
$$

The new question arises: how can we find the minimum of this functional? The answer is rather obvious and comes from physics, and is formulated according to the stationary action principle: the minimum of the functional appears at the argument $Q$ where its first variation (the Gâteaux derivative) vanishes. Calculating the first variation of $\Phi(K(Q))$ with respect to $Q$ under given $D, c_{p}, c_{t}$ and $c_{s}$, we get

$$
\delta \Phi(K(Q))=\partial_{K} \Phi(K) \partial_{Q} K(Q) \delta Q \text {. }
$$

The condition $\delta \Phi(K(Q))=0$ implies that

$$
\partial_{K} \Phi(K) \partial_{Q} K(Q) \delta Q=0
$$


for any variation $\delta Q$, where $\partial_{K} \Phi(K)$ and $\partial_{Q} K(Q)$ denote functional derivatives, and the argument $Q^{*}$, at which the product of these derivatives vanishes, gives us the solution to our optimization problem.

To illustrate this, let us consider a class of linear functionals given by (36). Let us denote the branches of the fuzzy number $K(Q)$ by $\left(f_{K}, g_{K}\right)$, and for the remaining quantities we will adapt the previous notation by using the appropriate subscripts, i.e.

$$
D=\left(f_{D}, g_{D}\right), Q=\left(f_{Q}, g_{Q}\right), c_{p}=\left(f_{p}, g_{p}\right), c_{t}=\left(f_{t}, g_{t}\right), c_{s}=\left(f_{s}, g_{s}\right) \text {. }
$$

We keep similar assumption on the discount functions $r(Q)$ and $s(Q)$ as two step functions with the steps represented by relationships (2) and (3), where $r_{i}$ and $s_{j}$ are here crisp $^{3}$, while the border values $Q_{i}^{r}, Q_{j}^{S}, i=1,2, j=1,2$, are Ordered Fuzzy Numbers, which satisfy the inequalities (10). Hence we can define 5 subinterval (11). The linear functional superposed on the fuzzy cost $K(Q)$ has the form of:

$$
\Phi(K(Q))=\Phi\left(f_{K}, g_{K}\right)=\int_{0}^{1} f_{K}(s) d h_{1}(s)+\int_{0}^{1} g_{K}(s) d h_{2}(s)
$$

where, due to (9) and the step functions $r(Q)$ and $s(Q)$, the pair of functions $f_{K}, g_{K}$ represents 6 pairs, namely

$$
\begin{gathered}
f_{K_{i j}}(s)= \\
=f_{p}(s)\left(1-r_{i}\right)\left(f_{D}(s)+R f_{Q}(s)\right)+f_{t}(s) \frac{f_{D}(s)}{f_{Q}(s)}+180 f_{s}(s)\left(1-s_{j}\right)\left(f_{Q}(s)+2 Q_{0}\right) \\
g_{K_{i j}}(s)= \\
=g_{p}(s)\left(1-r_{i}\right)\left(g_{D}(s)+R g_{Q}(s)\right)+g_{t}(s) \frac{g_{D}(s)}{g_{Q}(s)}+180 g_{s}(s)\left(1-s_{j}\right)\left(g_{Q}(s)+2 Q_{0}\right)
\end{gathered}
$$

where $i, j=0,1,2$. Like in Section 2 we can introduce new index $k=0,1,2,3,4$ and use the same identification as in (15) to decrease the number of pairs of functions $\left(f_{K_{i j}}, g_{K_{i j}}\right)$. Now, we take variation in (16) where the functional is given by (21), to

$$
\begin{gathered}
\delta \Phi(K(Q))=\int_{0}^{1}\left[f_{p}(s)\left(1-r_{i}\right) R-f_{t}(s) \frac{f_{D}(s)}{f_{Q}^{2}(s)}+180 f_{s}(s)\left(1-s_{j}\right)\right] \delta f_{Q}(s) d h_{1}(s)+ \\
\quad+\int_{0}^{1}\left[g_{p}(s)\left(1-r_{i}\right) R-g_{t}(s) \frac{g_{D}(s)}{g_{Q}^{2}(s)}+180 g_{s}(s)\left(1-s_{j}\right)\right] \delta g_{Q}(s) d h_{2}(s)
\end{gathered}
$$

with $i, j=0,1,2$. We could consider two cases:

Case A: The functions $h_{1}$ and $h_{2}$ are absolutely continuous, and

Case B: The functions $h_{1}$ and $h_{2}$ are singular, i.e. the derivatives $h_{1}^{\prime}(s)$ and $h_{2}^{\prime}(s)$ equal zero almost everywhere.

It is interesting to notice that in the first cases particular forms of $h_{1}$ and $h_{2}$ in (21) do not affect the optimal value of $Q$, it does affect, however, the optimal value of the crisp $\operatorname{cost} \Phi(K(Q))$. Hence we formulate remark concerning the first case.

Theorem 1. If the total inventory cost $K(Q)$ arising from fuzzy unit costs of delivery $c_{t}$, of inventory $c_{s}$, of the annual demand $D$, of the discount functions $r(Q)$ and $s(Q)$, and of the banking interest rate $R$, are given by (9) and the decision maker chooses the

\footnotetext{
${ }^{3}$ If there are also fuzzy numbers from $\mathcal{R}$, then final results will be of the same type with 4 extra pairs of functions appearing in a multiplicative way in the expressions for $f_{M_{k}}(s)$ and $g_{M_{k}}(s)$ from (26).
} 
defuzzification functional $\Phi$ in (21), then in Case A the economic order quantity is given by two phase optimization procedure:

- $\quad$ Phase 1. On each subinterval $L_{0}, L_{1}, L_{2}, L_{3}, L_{4}$ the optimal values are found

where

$$
q_{k}^{*}=\Phi\left(Q_{k}^{*}\right) \text {, with } Q_{k}^{*}=\left(f_{Q_{k}^{*}}, g_{Q_{k}^{*}}\right) \text {, }
$$

$$
f_{Q_{k}^{*}}=\sqrt{\frac{f_{t}(s) f_{D}(s)}{f_{M_{k}}(s)}}, g_{Q_{k}^{*}}=\sqrt{\frac{g_{t}(s) g_{D}(s)}{g_{M_{k}}(s)}}, s \in[0,1],
$$

with $f_{M_{k}}(s)=f_{p}(s)\left(1-r_{i}\right) R+180 f_{s}(s)\left(1-s_{j}\right)$, and expression for $g_{M_{k}}(s)$ is analogous. The notation and the identification between the indexes $k$ and $[i j]$ are coming from (15).

- Phase 2. From these five values $\Phi\left(Q_{k}^{*}\right), k=0,1,2,3,4$ and the values of the boundary numbers: $Q_{1}^{r}, Q_{1}^{S}, Q_{2}^{r}, Q_{2}^{S}, D$, the optimal value is calculated according to

$Q^{\text {opt }}=\arg \min \left\{\Phi\left(K\left(Q_{k}^{*}\right)\right), \Phi\left(K\left(Q_{1}^{r}\right)\right), \Phi\left(K\left(Q_{1}^{S}\right)\right), \Phi\left(K\left(Q_{2}^{r}\right)\right), \Phi\left(K\left(Q_{2}^{s}\right)\right), \Phi(K(D))\right\}$. In [Chwastyk, Kosiński, 2013] the authors have discussed a less complex case. On Fig. 4 the graph of the cost function (9) without any discount on storage inventory and the cost of frozen capital for different values of $Q$ is plotted. In the next subsection a numerical example will be presented.

\subsection{Numerical example}

In [Kuchta, 2001] the author considered the problem of minimizing the value of the fuzzy cost $K(Q)$ of a company in which

$$
K(Q)=D \cdot c+c_{t} \cdot \frac{D}{Q}+c_{s} \cdot \frac{Q}{2} .
$$

It corresponds to our problem by neglecting the cost of frozen capital, discount of purchase cost, discount stored inventory cost as well as the influence of safety stock related to daily consumption of inventory. Then the storage cost equals $K_{S}=c_{S} \cdot \frac{Q}{2}$ instead of (8). It corresponds to the case formulated as the optimization problem from Section 2.

Kuchta in her paper [Kuchta, 2001] considered first the crisp (deterministic) case with the following data: $D=1000, c=10, K_{t}=8$ and $K_{s}=7$. In her calculation the final economic order quantity $Q_{k}$ was 46 and the total cost $K\left(Q_{k}\right)$ corresponding to this order value was 10329 . In our calculation we get $Q_{k}=47.8$ and the cost value $K\left(Q_{k}\right)=10334.7$. These values are different from those of Kuchta in (Kuchta, 2001).

Then she considered the fuzzy case with the same crisp values of $D$ and $c$, but with the fuzzy transportation cost $\widetilde{K}_{t}$ represented by the triangular membership function $(7,8,9)$ and the fuzzy storage cost $\widetilde{K}_{S}$ represented by the triangular membership function $(1.5,7,15)$. Determination of the economic order quantity in that fuzzy case is not unique, and is based on some estimation to be done by the decision maker if he/she is supplied with a set of fuzzy cost value determined with the help of 
(28) in which the fuzzy values $\widetilde{K}_{t}$ and $\widetilde{K}_{s}$ appear, together with $2 M+1$ crisp values of $Q$ from the vicinity of $Q_{k}$, where $M$ is a natural number determined by the decision maker (in Kuchta's paper it was 50). Then the decision maker has to choose from those $2 M+1$ fuzzy cost values the one most suitable for him/her.

The same example will be considered here for OFN. Adapting our general solution formula (26) with vanishing discount functions we obtain

$$
f_{Q^{*}}(s)=\sqrt{\frac{2 \cdot f_{t}(s) \cdot f_{D}(s)}{f_{S}(s)}} \text { and } g_{Q^{*}}(s)=\sqrt{\frac{2 \cdot g_{t}(s) \cdot g_{D}(s)}{g_{s}(s)}} \text {. }
$$

In contrast to Kuchta's approach, if we apply our method and the linear defuzzification functional (24), then from the Theorem for the Case A (absolutely continuous $h_{1}$ and $h_{2}$ in (21)), we get the explicit expression of the fuzzy EOQ

and

$$
f_{K^{*}}(s)=f_{D}(s) \cdot c+f_{t}(s) \cdot \frac{f_{D}(s)}{f_{Q^{*}}(s)}+f_{s}(s) \cdot \frac{f_{Q^{*}}(s)}{2}
$$

$$
g_{K^{*}}(s)=g_{D}(s) \cdot c+g_{t}(s) \cdot \frac{g_{D}(s)}{g_{Q^{*}}(s)}+g_{s}(s) \cdot \frac{g_{Q^{*}}(s)}{2} .
$$

To this end let us choose the representation of two convex triangular fuzzy numbers $\widetilde{K}_{t}$ and $\widetilde{K}_{s}$ as Ordered Fuzzy Numbers. We know that to each CFN there are two corresponding OFNs, and they differ by orientation. Hence for $\widetilde{K}_{t}$ we have $(9-$ $s, 7+s)$ and $(7+s, 9-s)$, with $s \in[0,1]$ (Fig.2). On the other hand for $\widetilde{K}_{s}$ we have $(15-8 s, 1.5+5.5 s)$ and $(1.5+5.5 s, 15-8 s)$ (Fig.3). For $\widetilde{K}_{t}$, if we take the first OFN, which has the so-called negative orientation, it means that our estimation of future transportation cost is rather optimistic, the cost is at most around 8; on the other hand if we take the second OFN, namely $(9-s, 7+s)$, then we are rather pessimistic: the transportation cost is at least around 8 .

For further calculation we assume the optimistic viewpoint and take for $f_{t}(s)=9-$ $s$ and $g_{t}(s)=7+s$, while for $f_{s}(s)=15-8 s$ and $g_{s}(s)=1.5+5.5 s$. Please note that we could assume 3 different cases and, consequently, 3 different solutions for fuzzy EOQ could arise.

Applying the formula appearing in $\operatorname{Remark}^{4}$, with $f_{D}(s)=1000, f_{M}(s)=f_{S}(s) / 2$ and $g_{D}(s)=1000, g_{M}(s)=g_{s}(s) / 2$ we obtain the fuzzy EOQ as the Ordered Fuzzy Number

$$
f_{Q^{*}}(s)=\sqrt{\frac{2000 \cdot(9-s)}{15-8 s}}, \quad g_{Q^{*}}(s)=\sqrt{\frac{2000 \cdot(7+s)}{1.5+5.5 s}}, \quad s \in[0,1] .
$$

\footnotetext{
${ }^{4}$ Notice that in our example $D$ is crisp and is represented by the pair of constant functions $\left(1000^{\circ}, 1000\right)$
} 
FIGURE 2.

\section{OFN of fuzzy transportation cost}
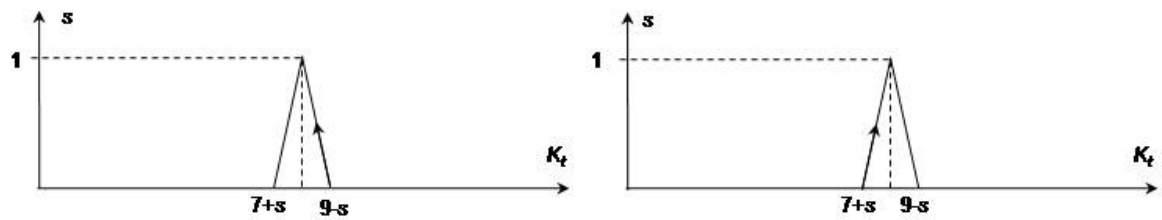

Source: own work.

FIGURE 3.

\section{OFN of fuzzy storage cost}
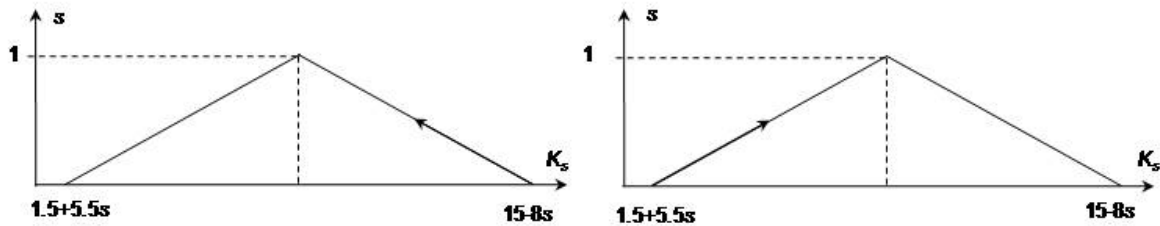

Source: own work.

From the last expression we could easily calculate the fuzzy minimal inventory cost $K\left(Q^{*}\right)$. Please note that neither $Q^{*}$ nor $K\left(Q^{*}\right)$ can be represented in the form of CFN with triangular membership function. We could draw figures for them by substituting values of $s$ from $[0,1]$ interval. By applying a particular defuzzification functional we could calculate the crisp values corresponding to $Q^{*}$ and $K\left(Q^{*}\right)$. At the end of this section we point out the characteristic values of $Q^{*}$, namely

$$
f_{Q^{*}}(0)=34.6, f_{Q^{*}}(1)=g_{Q^{*}}(1)=47.8, g_{Q^{*}}(0)=96.6 \text {. }
$$

Please note that by applying of the defuzzification functional $\phi=\phi_{M O M}$ to $Q^{*}$ we obtain the crisp EOQ $\phi_{M O M}\left(Q^{*}\right)=f_{Q^{*}}(1)=47.8$, which is equal to $Q_{k}$ from the deterministic case. Corresponding to those values, the characteristic values of the cost are:

$$
\begin{gathered}
f_{K^{*}}(0)=10000+f_{t}(0) \cdot \frac{1000}{f_{Q^{*}(0)}}+f_{s}(0) \cdot \frac{f_{Q^{*}}(0)}{2}=10519.6, \\
f_{K}^{*}(1)=g_{K}^{*}(1)=10000+f_{t}(1) \cdot \frac{1000}{f_{Q^{*}(1)}}+f_{s}(1) \cdot \frac{f_{Q^{*}(1)}}{2}=10334.6, \\
g_{K}^{*}(0)=10000+g_{t}(0) \cdot \frac{1000}{g_{Q^{*}}(0)}+g_{s}(0) \cdot \frac{g_{Q^{*}(0)}}{2}=10144.9 .
\end{gathered}
$$

If we look at the fuzzy cost values in [Kuchta, 2001], we can see in Table 7.1 on page 112 that domains of triangular membership functions of those values vary from 10138 to 10513. Moreover, fuzzy values of cost are related to the range of order quantities from 91 to 36 . In our calculation this range is 34.6 to 96.6. 


\section{Optimal orders under dynamic conditions}

In the next subsection we file a solution to the deterministic optimization problem with the help of a controller. In any company material demand from warehouse during the production processes is often irregular. It is related to uneven production or technical problems. Thus, the inventory optimization problem becomes more complicated in relation to the order quantity and the frequency of orders, as well as transportation costs. The algorithm described here solves that problem in a relatively easy way by some simple algebraic operations. The final result is a controller.

In order to always provide a proper state of inventory quantity in a warehouse, one has to provide appropriate supplies. Each supply requires us to determine an optimal order quantity which should be calculated for a given time period $t$ taking into account the actual status of warehouse inventory while minimizing the costs of purchase, transportation and maintaining items in the warehouse.

In our solution we keep the previous notations, adding some new variables. Moreover, some new assumptions are also made.

1. Demands are not constant in time and depend on the length of time period for which the order is made.

2. Sales is not uniform and hence actual status of the warehouse is different in time.

3. The next delivery arrives after a known period of time that may change by $\pm \delta t$.

4. The safety stock depends on the length of period of time for which the order is made.

5. Cost of transportation depends on the order quantity.

6. There is a discount on purchase.

7. The partitioning of domains of variables does not have to be regular.

For presenting the solution we use the following additional variables (cf. Section 2):

- $\quad M-$ actual inventory status in the warehouse;

- $\quad q$ - possible order quantity used for calculations;

- $\quad \Delta Q$ - a value by which $Q$ (or $q$ ) might be increased or decreased;

- $\quad t$ - time period for which particular order is made, its value may be changed by some rules of our controller;

- $\Delta t$ - time period by which $t$ may be increased or decreased, or by its multiplicity;

- $\quad D(t)$ - inventory demand for period $t$, (with safety stock included);

- $\theta(t)$ - safety stock;

- $\quad r(q)$ - discount function on purchase;

- $\quad K_{t}(q)$ - transportation cost function, in general nonlinear, e.g. a step function;

- $\quad P$ - a set of possible order quantities located on discontinuities of $K(q)$ function, related to the step characteristics of the discount function $r(q)$ and the transportation cost functions $K_{t}(q)$. 


\subsection{Total cost evaluation}

If $q$ represents a possible order quantity, then the total cost is

$$
K(q)=K_{p}(q)+K_{t}(q)+K_{s}(q) .
$$

Assuming that the discount function on purchase $r(q)$ depending on the amount of $q$ is a step functions, and its form is similar to that $r(q)$ from section 2, with the small change of $Q_{1}^{r}, Q_{2}^{r}$ into $q_{1}^{r}, q_{2}^{r}$, respectively. The cost of transportation (delivery) forms a step function, as well:

$$
K_{t}(q)=\left\{\begin{array}{ccc}
K_{0}=0 & \text { if } & 0<q<q_{1}^{t} \\
K_{1} & \text { if } & q_{1}^{t} \leq q<q_{2}^{t} \\
K_{2} & \text { if } & q_{2}^{t} \leq q \leq D
\end{array}\right.
$$

where $q_{1}^{r}, q_{2}^{r}, q_{1}^{t}$ and $q_{2}^{t}$ are fixed amounts of the item's quantity (here 3 steps have been assumed, however, more or fewer steps can be also considered). The purchase cost $K_{p}(q)$ depends on the discount $r(q)$ and the unit price $c_{p}$ as below:

$$
K_{p}(q)=c_{p} \cdot(1-r(q)) \cdot q
$$

Due to discontinuity of both functions $r(q)$ and $K_{t}(q)$ as well as the quantized nature of $q$ in deliveries ${ }^{5}$ the order quantity $Q$ cannot be an arbitrary number: it needs to be adjusted even if the economic order quantity $Q^{*}$ has been calculated by solving an appropriate optimization problem. Hence deliveries can be partitioned by $\Delta Q$ and the other relevant functions: $\theta(t)$ and $D(t)$. Please note that arguments at which the function $r(q)$ is discontinuoued may be different from those of the function $K_{t}(q)$. Hence we formulate two rules which form the basis rule of our controller.

1. IF $t_{\text {old }}$ is the time period for which inventory demand is made THEN the new time $t_{\text {new }}$ is equal to $t-\Delta t$ if the state $M$ is lower than the safety stock $\theta(t)$, or is equal to $t+\Delta t$, providing that the state $M$ is higher than the demand $D(t)$, otherwise $t_{\text {new }}=t_{\text {old }}$.

2. IF $P$ is the set of discontinuity arguments of both functions $r(q)$ and $K_{t}(q)$ THEN the final order quantity $Q$ is the smallest element of $P$ that with the current amount in the warehouse would suffice for the next period of time with the minimal cost. IF such an element of $P$ does not exist, THEN the final order quantity should be equal to the smallest multiple of $\Delta Q$, that with the current amount in the warehouse would suffice for the next period of time.

The above rules need explicit relationships to be applied in practice. We assume further that $K_{S}(q)$ is constant. Hence for the first rule

$$
t_{\text {new }}\left(t_{\text {old }}, M\right)=\left\{\begin{array}{ccc}
t_{\text {old }}-\Delta t & \text { if } & M<\theta\left(t_{\text {old }}\right) \\
t_{\text {old }} & \text { if } & \theta\left(t_{\text {old }}\right)<M<D\left(t_{\text {old }}\right) . \\
t_{\text {old }}+\Delta t & \text { if } & D\left(t_{\text {old }}\right)<M
\end{array}\right.
$$

\footnotetext{
${ }^{5}$ If the item to be ordered is coal, it is impossible to buy a fraction of tones; in practice coal is bought in full tons or in full boxcars.
} 
For the second rule we will use the notation $[\cdot]$ and $[\cdot]$ in order to keep the quantized nature of $q$. Hence the optimal order quantity $Q^{*}$ is the function of $M$ and $D\left(t_{\text {new }}\right)$ and is equal to $q^{*}$ if such $q^{*}$ exists which is equal to

$$
\operatorname{argmin}\left\{K(q):\left[D\left(t_{\text {new }}\right)-\lfloor M\rfloor\right] \leq q \wedge q \in P \wedge K(q) \leq K\left(\left[D\left(t_{\text {new }}\right)-\lfloor M\rfloor\right\rceil\right)\right\}
$$

and otherwise

$$
Q^{*}=\left\lceil D\left(t_{\text {new }}\right)-M\right\rceil .
$$

In the next subsection a numerical example will be presented.

\subsection{Numerical example}

Let us consider an example of an item measured in number of units, with 8000 unit as the base quantity, and with the following data: $c_{p}=10, c_{s}=7, M=0, \Delta Q=5000$, $D(t)=t \cdot 8000 \cdot 110 \%, \Delta t=1$ week, $\theta(t)=D(t) \cdot 10 \% / 110 \%, K_{t}(q)=$ 7000 and

$$
r(q)=\left\{\begin{array}{ccc}
0 \% & \text { if } & 0<q<5000 \\
5 \% & \text { if } & 5000 \leq q<10000 \\
10 \% & \text { if } & 10000 \leq q<20000 \\
15 \% & \text { if } & q \geq 20000
\end{array} .\right.
$$

In general, the graph $K(q)$ on Fig.4 does not have to be linear between border values that are not multiples of $\Delta Q$. However, those values are not achievable as an order quantity, hence there is no need to consider them. The circled areas are interesting because of the optimization possibility. Those values of $q$ belong to the set $P$. In the interval $(4000 ; 4999]$ the cost $K(q)$ is in $(61000 ; 74486,5]$, measured in PLN. But in the next interval with the bigger order, the value $K(5000)=71987$ PLN appears, which is smaller than the upper limit of the previous interval. It means that ordering bigger amount means that cost will be the same or lower. So it is better to buy more, in this case 5000 units. Thus, the knowledge base can be changed in those particular places.

In Table 1, the results for $q$ are presented. If $t$ is changed, we have to order our item not for the old value of $t$ but for the new one, according to (31). This is presented in Table 2 together with the final results of the controller for $Q$.

FIGURE 4.

The graph of $K(q)$ of the controller.

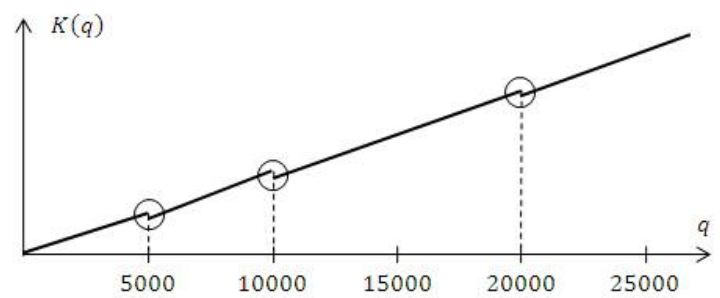

Source: own work. 
TABLE 1.

Partitioning of $q$ based on different factors together with the rules for $q$

\begin{tabular}{|c|c|c|c|c|c|}
\hline \multicolumn{2}{|c|}{$\boldsymbol{q}$} & \multicolumn{4}{|c|}{$\boldsymbol{t}$} \\
\cline { 3 - 6 } & $\mathbf{1}$ & $\mathbf{2}$ & $\mathbf{3}$ & $\mathbf{4}$ \\
\hline \multirow{4}{*}{$\boldsymbol{M}$} & $0-799$ & 10000 & 20000 & 30000 & 40000 \\
\cline { 2 - 6 } & $800-1599$ & 10000 & 20000 & 30000 & 35000 \\
\cline { 2 - 6 } & $1600-2399$ & 10000 & 20000 & 25000 & 35000 \\
\cline { 2 - 6 } & $2400-8799$ & 10000 & 20000 & 25000 & 35000 \\
\cline { 2 - 6 } & $8800-17599$ & 0 & 10000 & 20000 & 30000 \\
\cline { 2 - 6 } & $17600-26399$ & 0 & 0 & 10000 & 20000 \\
\cline { 2 - 6 } & $26400-\ldots$ & 0 & 0 & 0 & 10000 \\
\hline
\end{tabular}

Source: own work.

TABLE 2.

The knowledge base for changing $t$ together with the final results of the controller

\begin{tabular}{|c|c|c|c|c|c|}
\hline \multicolumn{2}{|c|}{$t_{\text {new }}$} & \multicolumn{4}{|c|}{$t_{\text {old }}$} \\
\cline { 2 - 6 } & $\mathbf{1}$ & $\mathbf{2}$ & $\mathbf{3}$ & 4 \\
\hline \multirow{4}{*}{$\boldsymbol{M}$} & $0-799$ & $t_{\text {old }}$ & $t_{\text {old }}-\Delta t$ & $t_{\text {old }}-\Delta t$ & $t_{\text {old }}-\Delta t$ \\
\cline { 2 - 6 } & $800-1599$ & $t_{\text {old }}$ & $t_{\text {old }}-\Delta t$ & $t_{\text {old }}-\Delta t$ & $t_{\text {old }}-\Delta t$ \\
\cline { 2 - 6 } & $1600-2399$ & $t_{\text {old }}$ & $t_{\text {old }}$ & $t_{\text {old }}-\Delta t$ & $t_{\text {old }}-\Delta t$ \\
\cline { 2 - 6 } & $2400-8799$ & $t_{\text {old }}$ & $t_{\text {old }}$ & $t_{\text {old }}$ & $t_{\text {old }}-\Delta t$ \\
\cline { 2 - 6 } & $8800-17599$ & $t_{\text {old }}+\Delta t$ & $t_{\text {old }}$ & $t_{\text {old }}$ & $t_{\text {old }}$ \\
\cline { 2 - 6 } & $17600-26399$ & $t_{\text {old }}+\Delta t$ & $t_{\text {old }}+\Delta t$ & $t_{\text {old }}$ & $t_{\text {old }}$ \\
\cline { 2 - 6 } & $26400-\ldots$ & $t_{\text {old }}+\Delta t$ & $t_{\text {old }}+\Delta t$ & $t_{\text {old }}+\Delta t$ & $t_{\text {old }}$ \\
\hline
\end{tabular}

\begin{tabular}{|c|c|c|c|c|c|}
\hline \multicolumn{2}{|c|}{$\boldsymbol{Q}$} & \multicolumn{4}{|c|}{$\boldsymbol{~}$} \\
\cline { 3 - 6 } & $0-799$ & 1 & $\mathbf{2}$ & $\mathbf{3}$ & $\mathbf{4}$ \\
\hline \multirow{4}{*}{$\boldsymbol{M}$} & $800-1599$ & 10000 & 10000 & 20000 & 40000 \\
\cline { 2 - 6 } & $1600-2399$ & 10000 & 10000 & 20000 & 30000 \\
\cline { 2 - 6 } & $2400-8799$ & 10000 & 10000 & 25000 & 25000 \\
\cline { 2 - 6 } & $8800-17599$ & 10000 & 10000 & 20000 & 30000 \\
\cline { 2 - 6 } & $17600-26399$ & 0 & 10000 & 10000 & 20000 \\
\cline { 2 - 6 } & $26400-\ldots$ & 0 & 0 & 10000 & 10000 \\
\hline
\end{tabular}

Source: own work.

Let us start with $M=0, t_{\text {old }}=4$. We need 30000 items and have to decrease $t$ by a week i.e. $t_{\text {new }}=3$. After 3 weeks we are left with 3600 units in the warehouse, so the next order should consist of 25000 pieces and $t=3$. Now we are left with 2200 units in 
the warehouse, and consequently, the next order should consist of 20000 pieces, and $t=$ 2. After 2 weeks we are left with 4600 units and the next order should consist of 20000 and $t=2$, and so on. If there are no bigger fluctuations in demand, the controller should give each time the same or similar results throughout the whole year.

\section{Conclusions}

We have solved a problem originating from management of inventory, using the setup of Ordered Fuzzy Numbers (OFN), and demonstrated its applicability in modelling of the influence of imprecise quantities and preferences of a decision maker. Thanks to welldefined arithmetic of OFN one can construct an efficient decision support tool when data are imprecise. In Section 2 we introduced economic order quantity model and in the next section its fuzzy solution. In Section 4 of the paper we have introduced some dynamics in management of inventory and showed that in a simplest case a rule based controller can play a role of an optimizing tool. The future work can be connected to the extension of the economic order quantity model and reflect its reality.

\section{Bibliography}

Chwastyk A., Kosiński W. 2013 Fur:y Calculus with Applications, "Mathematica Applicanda - Matematyka Stosowana", 41(1) (on line).

Goetschel R. Jr., Voxman W. 1986 Elementary Fuzzy Calculus, "Fuzzy Sets and Systems", 18(1).

Kosiński W.K., Kosiński W., Kościeński K. 2013 Ordered Fuzay Numbers Approach to an Investment Project Evaluation, "Management and Production Engineering Review", 4(2).

Kosiński W., Prokopowicz P., Ślęzak D. 2002 Fuz:yy Numbers with Algebraic Operations: Algorithmic Approach, [in:] Proc.IIS'2002, (eds.) M. Klopotek, S. T. Wierzchoń, M. Michalewicz M., Sopot.

Kosiński W., Prokopowicz P., Ślęzak D. 2003 Ordered Fuzzy Numbers, „Bulletin of the Polish Academy of Sciences", 51 (3).

Kosiński W. 2006 On Fuz:y Number Calculus, "Int. J. Appl. Math. Comput. Sci.”, 16. Kuchta D. 2001 Mieke.ea matematyka w zarzadzaniu. Zastosowanie liczb przedziatowych $i$ rozmytych $w$ rachunkowości zarzqdczej, Wrocław.

Nguyen H.T. 1978 A Note on the Extension Principle for Fuz:y Sets, "J. Math. Anal. Appl”, 64.

Vuješević M., Petrović D., Petrović R., (1996), EOQ Formula when Inventory Cost is Fuzzy, "Int. J. Production Economics", 45.

\section{Appendix}

Proposed recently by Kosiński and his two co-workers Prokopowicz and Ślęzak [Kosiński et al., 2002, Kosiński et al., 2003, Kosiński, 2006] an extended model of Convex 
Fuzzy Numbers (CFN) [Nguyen, 1978], called Ordered Fuzzy Numbers (OFN), does not require any existence of membership functions. In this model we can see an extension of CFN - model, when one takes a parametric representation of fuzzy numbers know since 1986, [Goetschel, Voxman, 1986] of convex fuzzy numbers.

Definition 1. By an Ordered Fuzzy Number we understand a pair of functions $(f, g)$ defined on the unit interval $[0,1]$, which are continuous functions (or of bounded variations) [Kosiński et al., 2002; Kosiński et al., 2003, Kosiński, 2006].

On OFN, denoted by $\mathcal{R}$ (or $\mathcal{R}_{B V}$ ), four algebraic operations have been proposed between fuzzy numbers and crisp (real) numbers, in which component wise operations are present. In particular

$$
f_{C}(y)=f_{A}(y) * f_{B}(y), \quad g_{C}(y)=g_{A}(y) * g_{B}(y),
$$

where "* " works for" + ", " - ", " . " and " $\div$ ", respectively, and where $A \div B$ is defined, if the functions $\left|f_{A}\right|$ and $\left|f_{B}\right|$ are bounded from below. Hence any fuzzy algebraic equation $A+X=C$ with $A$ and $C$ as OFN possesses a solution.

A relation of partial ordering in the space of all OFN, can be introduced by defining the subset of 'positive' Ordered Fuzzy Numbers: a number $A=(f, g)$ is not less than zero, and by writing

$$
A \geq 0 \text { iff } f \geq 0, g \geq 0 .
$$

In this way the set $\mathcal{R}$ (or $\mathcal{R}_{B V}$ ) becomes a partially ordered ring. Note, that for each two fuzzy numbers $A=\left(f_{A}, g_{A}\right), B=\left(f_{B}, g_{B}\right)$ as above, we may define $A \wedge B=: F$ and $A \vee B=: G$, both from $\mathcal{R}$, by the relations: $F=\left(f_{F}, g_{F}\right)$, if $f_{F}=\inf \left(f_{A}, f_{B}\right)$, $g_{F}=\inf \left(g_{A}, g_{B}\right)$. Similarly, we define $G=A \vee B$ and we get the next structure on $\mathcal{R}$, namely a lattice. Its sublattice will be a chain of real numbers. If $A \leq B$, then the set $[A, B]=\{C \in \mathcal{R}: A \leq C \leq B\}$ will be a sublattice of the lattice $(\mathcal{R}, \leq)$.

In dealing with applications of fuzzy numbers we need set of functionals that map each fuzzy number into real, and in a way that is consistent with operations on reals. Those operations are called defuzzifications. To be more strict we introduce.

Definition 2. A map $\Phi$ from the space $\mathcal{R}$ (or $\mathcal{R}_{B V}$ ) of all OFN's to reals is called a defuzzification functional if is satisfies:

$$
\begin{array}{ll}
- & \Phi\left(c^{*}\right)=c, \\
- & \Phi\left(A+c^{*}\right)=\Phi(A)+c, \\
- & \Phi(c A)=c \Phi(A) \text { for any } c \in R \text { and } A \in \mathcal{R}, \\
- & \Phi(A) \geq 0 \text { if } A \geq 0
\end{array}
$$

where $c^{*}(s)=\left(c^{\prime}, c^{\prime}\right)$, where $c^{\prime}(s)=c$ for any $s \in[0,1]$, represents crisp number (a real) $c \in R$

The linear functionals, as MOM (middle of maximum), FOM (first of maximum), LOM (last of maximum) are given by specification of $h_{1}$ and $h_{2}$

$$
\Phi\left(f_{A}, g_{A}\right)=\int_{0}^{1} f_{A}(s) d h_{1}(s)+\int_{0}^{1} g_{A}(s) d h_{2}(s),
$$

where $h_{1}, h_{2}$ are nonnegative functions of bounded variation and

$$
\int_{0}^{1} d h_{1}(s)+\int_{0}^{1} d h_{2}(s)=1 .
$$


If we substitute $h_{1}(s)$ and $h_{2}(s)$ by $\lambda H(s)$ and $(1-\lambda) H(s)$, respectively, where $0 \leq \lambda \leq 1$, and $H(s)$ is the step Heaviside function (with the step at $s=1$ ), we may obtain all the classical linear defuzzification functionals known for the fuzzy numbers of Zadeh, namely: MOM (middle of maxima), FOM (first of maximum), LOM (last of maximum) and RCOM (random choice of maximum), depending on the choice of $\lambda$; for example if for $h_{1}(s)$ and $h_{2}(s)$ we substitute $\frac{1}{2} H(s)$, then we get MOM. 